Doug Geisler, Eva K. Grebel, and Dante Minniti, eds.

\title{
Broad-band Photometric Evolution of Star Clusters
}

\author{
Léo Girardi \\ Dipartimento di Astronomia, Università di Padova \\ Vicolo dell'Osservatorio 5, I-35122 Padova, Italy \\ Max-Planck-Institut für Astrophysik, Karl-Schwarzschild-Str. 1, \\ D-85741 Garching bei München, Germany
}

\begin{abstract}
I briefly introduce a database of models that describe the evolution of star clusters in several broad-band photometric systems. Models are based on the latest Padova stellar evolutionary tracks - now including the $\alpha$-enhanced case and improved AGB models - and a revised library of synthetic spectra from model atmospheres. As of today, we have revised isochrones in Johnson-Cousins-Glass, HST/WFPC2, HST/NICMOS, Thuan-Gunn, and Washington systems. Several other filter sets are included in a preliminary way, like those used by the EIS and SDSS projects. The database contains also integrated magnitudes of single-burst stellar populations and Monte-Carlo simulations that show the stochastic dispersion of the colours as a function of cluster mass, age, and metallicity. The models are useful for several kinds of studies, including estimates of masses and ages of extragalactic star clusters observed by means of broad-band photometry.
\end{abstract}

\section{Introduction}

Broad-band magnitudes and colours are the primary source of information about the masses and ages of star clusters outside the Local Group. Up to recently, this was true even for the relatively nearby Magellanic Cloud (MC) clusters.

The simplest method of estimating ages and masses comes from the direct comparison of the observed colours and magnitudes with those predicted by population synthesis models: Two-colour diagrams may be used to assign an approximate age to a cluster; then, the integrated magnitude can be converted into a mass by means of the mass-to-light ratio predicted for that age. Of course, using models with complete and (as far as possible) updated physical prescriptions is mandatory in this process. However, even with the best models one should keep in mind that:

- Even for a given value of metallicity and age, cluster-to-cluster variations in the number/distribution of evolved stars lead to non-neglegible stochastic fluctuations in the colours and hence to errors in the estimated ages.

- Model colours may present systematic off-sets of some few hundredths of a magnitude, due e.g. to the use of filter pass-bands different from those used to collect the data. 

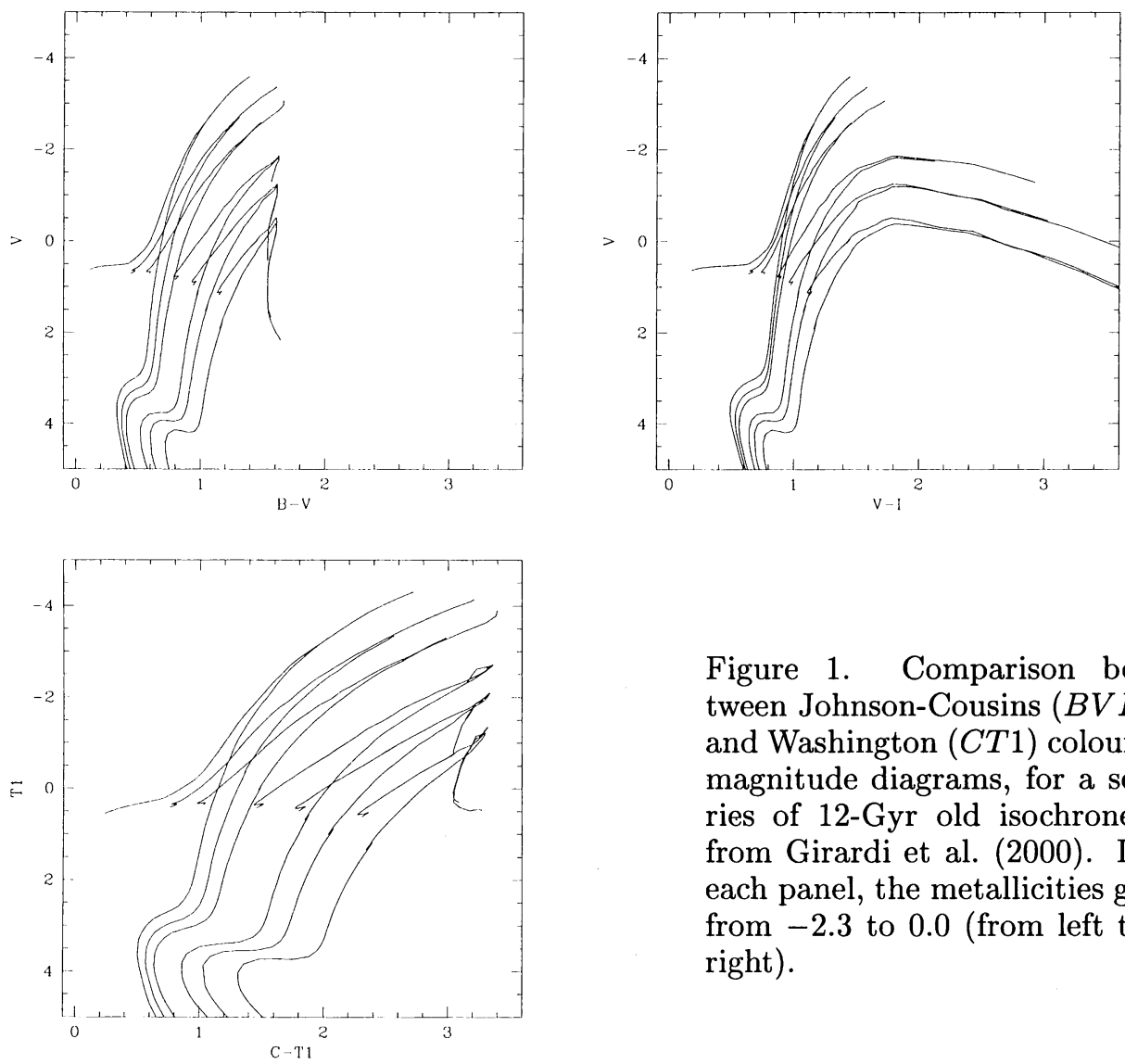

Figure 1. Comparison between Johnson-Cousins $(B V I)$ and Washington $(C T 1)$ colourmagnitude diagrams, for a series of 12-Gyr old isochrones from Girardi et al. (2000). In each panel, the metallicities go from -2.3 to 0.0 (from left to right).

- Some filter sets are expected to be either (i) better in separating clusters according to their age and metallicity, or (ii) less sensitive to the potential errors in the models, and shall hence be preferred.

- The mass-to-light ratio is determined, primarily, by the choice of the IMF and of its normalisation. Without a convenient check of this normalisation, model mass-to-light ratios (and mass estimates) are meaningless.

We are producing theoretical tools that can be used to face these problems. This contribution gives a very short anticipation about them.

\section{Isochrones in several photometric systems}

Our "basic set" of stellar evolutionary tracks and isochrones is presently composed by the Girardi et al. (2000) tracks for low- and intermediate-mass stars, complemented with Bertelli et al. (1994, and references therein) for the massive stars. For some metallicity values, we have the possibility of including the 
more sophisticated models for the TP-AGB phase from Marigo (2001, and references therein; Marigo \& Girardi 2001), or the extensive sets of tracks with an enhanced partition of $\alpha$-elements from Salasnich et al. (2000). Some nonovershooting isochrones are available, allowing a comparison with other classical models from the literature. Aditionally, we now have new sets with $Z=0.0001$ (yet unpublished) and $Z=0$ (Marigo et al. 2001).

We are now transforming these isochrone sets to several broad-band photometric systems (Girardi et al. 2001). To do so, we put together an extended library of stellar instrinsic spectra, which is based essentially on the synthetic atmospheres from Bessell et al. (1998) and Chabrier et al. (2000), and on the empirical data for M-giants from Fluks et al. (1994). Then, we perform synthetic photometry in several broad-band filter systems, and check for the zero-points. The resulting tables of bolometric corrections are used to transform our isochrone sets to observational quantities.

The process has been concluded for the Johnson-Cousins-Glass, Washington, Thuan-Gunn, HST/WFPC2 and HST/NICMOS systems, and for the filter sets used by the ESO Imaging Survey (i.e. EMMI, SOFI, and WFI cameras). The same is to be done for the Sloan Digital Sky Survey system, and others. In all cases, the only time-consuming task is the checking of zero-points. Given enough information (filter+detector transmission curves and zero-point definition), we are able to provide the same isochrones in any photometric system upon request.

In Fig. 1 we provide one example of the utility of this database: We compare the same set of isochrones of varying metallicity, in the Johnson-Cousins $B-V$ and $V-I$ colours, and in the Washington $C-T 1$ one. It is evident that $C-T 1$ provides the best separation (at least two times larger than in $B-V$ ) in metallicity over the complete magnitude interval, from the main sequence up to the upper RGB. And, importantly, all these filter pairs have similar throughputs. This confirms that the integrated $C-T 1$ colour is an excellent metallicity indicator for old clusters, in accordance with Geisler \& Sarajedini (1999). We did not find a comparable performance in other broad-band colours.

\section{Simulations of star clusters}

Once we assume an initial mass function (IMF), we can easily compute either (1) the integrated magnitudes for single-burst stellar populations, or (2) MonteCarlo simulations of clusters of given age, metallicity, and mass (or luminosity). Since the number ratio of stars above and below $1 M_{\odot}$ determines, in practice, the mass-to-light ratios in the models, the IMF should be properly normalized and calibrated in order to give reasonable values to this quantity. For a Salpeter IMF, a suitable low-mass cut-off has to be assumed (see Girardi \& Bica 1993; Girardi et al. 1995). If we use the more recent Kroupa (2001) IMF, $M / L_{V}$ come out already comparable to those observed in young LMC clusters, without the need of imposing a low-mass cut-off.

Together with the isochrones, we intend to provide the tools for simulating the integrated light of star clusters, for any assumed IMF, and as a function of age, metallicity, and total mass (or total luminosity). Needless to say, other effects such as reddening and photometric errors can be simulated as well. Such 

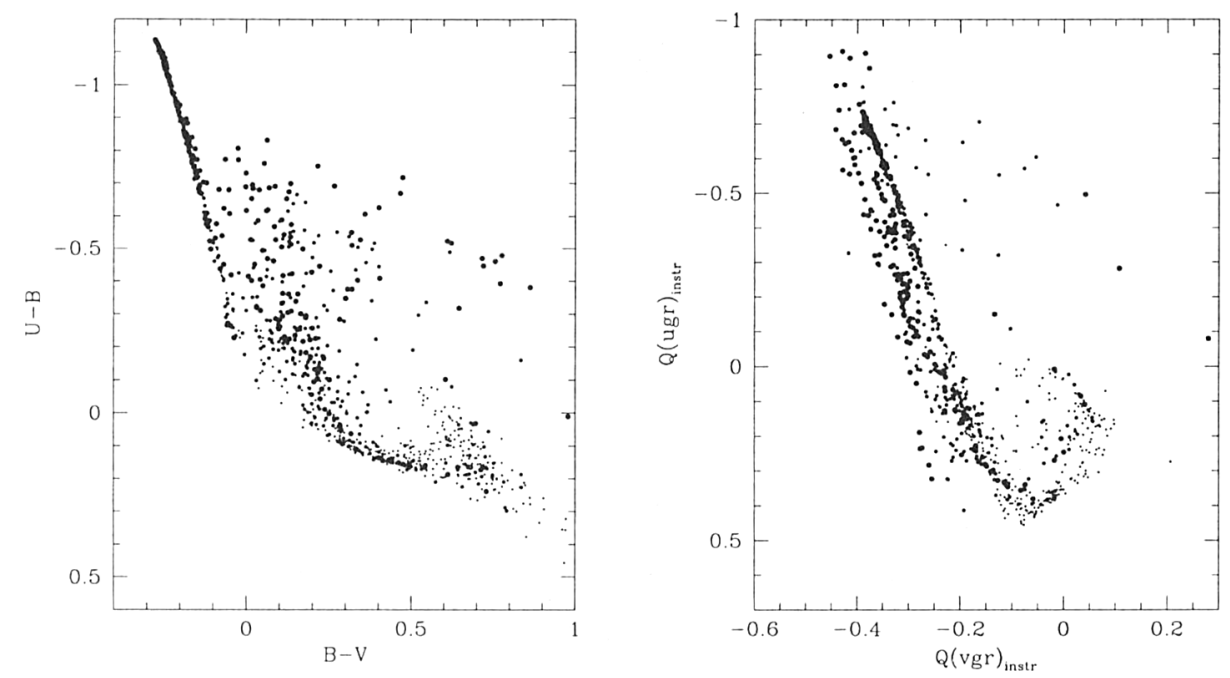

Figure 2. Simulations of a cluster population that obeys a $M^{-2}$ distribution of initial masses, a $t^{-1}$ distribution of ages, a linear $[\mathrm{Fe} / \mathrm{H}](t)$ age-metallicity relation, with 600 clusters above the assumed $M_{V}=5$ limiting magnitude. In the left panel, we have the $U B V$ plane of Johnson photometry, in the right one the $Q(u g r)-Q(v g r)$ diagram of ThuanGunn. See the text for details.

simulations show the natural colour (and magnitude) dispersion that derives from stochastic variations in the number of evolved stars, and are obviously useful for the interpretation of extragalactic cluster data.

Examples of cluster simulations are presented in the panels of Fig.2. They show a synthetic cluster population intended to mimic the LMC one, but obeying very simple distributions of their properties: we adopt a $M^{-2}$ distribution of initial masses, a $t^{-1}$ distribution of ages, a linear $[\mathrm{Fe} / \mathrm{H}](t)$ age-metallicity relation, with 600 clusters above the assumed $M_{V}=5$ limiting magnitude. The results are plotted both in the $U-B$ vs. $B-V$ plane of Johnson photometry (left panel), and in the $Q(u g r)-Q(v g r)$ diagram of Thuan-Gunn (right one). We recall that these two colour-colour diagrams became classical in the interpretation of LMC cluster data: the first one defines the Elson \& Fall (1985) age-dating method (see also Girardi et al. 1995), whereas the second one defines the SWB (Searle et al. 1980) classification scheme.

Although the general distribution of simulated points is similar to the observed ones for LMC clusters (see the counterparts in Bica et al. 1996, and Searle et al. 1980), a detailed comparison reveals that the assumed distributions are inappropriate. In particular, the age distribution $\propto t^{-1}$ causes an excess of clusters in some regions of the 2-colour planes. In this case, a detailed comparison with Bica et al. (1996) data would allow the derivation of a more realistic age distribution function (see Girardi et al. 1995 for an alternative approach).

Part of the models here mentioned are already available, in a preliminary form, at http://pleiadi.pd.astro.it/ ${ }^{\prime}$ girardi/faq.html. We are now 
working on a user-friendly interface, that would accompany the release of the complete database. All details are provided in forthcoming papers (starting with Girardi et al. 2001).

Acknowledgments. I greatly acknowledge the LOC for the travel grant that allowed me to attend this IAU Symp. Many thanks are due to G. Bertelli, A. Bressan, C. Chiosi, M. Groenewegen, P. Marigo, B. Salasnich, R. Tantalo and A. Weiss, my collaborators in the isochrone work, and to E. Bica, D. Geisler, E. Grebel, J. Holtzman for useful conversations. This work is funded mainly by the Italian MURST. The text was written during a stay at MPA supported by the TMR grant ERBFMRXCT960086.

\section{References}

Bertelli G., Bressan A., Chiosi C., Fagotto F, Nasi E., 1994, A\&AS, 106, 275

Bessell M.S., Castelli F., Plez B., 1998, A\&A 333, 231

Bica E., Clariá J.J., Dottori H., Santos Jr. J.F.C., Piatti A., 1996, ApJS 102, 57

Chabrier G., Baraffe I., Allard F, Hauschildt P., 2000, ApJ 542, 464

Elson R.A.W., Fall S.M., 1985, ApJ 299, 211

Fluks M.A., Plez B., The P.S., de Winter D., Westerlund B.E., Steenman H.C., 1994, A\&AS 105, 311

Geisler D., Sarajedini A., 1999, AJ 117, 308

Girardi L., Bica., 1993, A\&A 274, 279

Girardi L., Chiosi C., Bertelli G., Bressan A., 1995, A\&A, 298, 87

Girardi L., Bressan A., Bertelli G., Chiosi C., 2000, A\&AS 141, 371

Girardi L., Bertelli G., Bressan A., Chiosi C., Groenewegen M., Marigo P., Salasnich B., Tantalo R., Weiss A., 2001, in preparation

Kroupa P., 2001, MNRAS 322, 231

Marigo P., 2001, A\&A, 370, 194

Marigo P., Girardi L., 2001, A\&A submitted

Marigo P., Girardi L., Chiosi C., Wood P.R., 2001, A\&A 371, 152

Salasnich B., Girardi L., Weiss A., Chiosi C., 2000, A\&A 361, 1023

Searle L., Wilkinson A., Bagnuolo W.G., 1980, ApJ 239, 803 